\title{
Graphene films as transparent electrodes for photovoltaic devices based on cadmium sulfide thin films
}

\author{
Madhav Gautam, Zhengqi Shi, and Ahalapitiya H. Jayatissa* \\ Mechanical, Industrial, and Manufacturing Engineering Department \\ The University of Toledo, $\mathrm{OH} 43606$, USA.
}

\begin{abstract}
Graphene films were synthesized and characterized to demonstrate their potential applications as the transparent electrodes in photovoltaic devices. Graphene films were prepared by chemical vapor deposition of graphene on copper substrates followed by transferring them onto the desired substrates. The quality and uniformity graphene transferred onto different substrates were characterized by Raman spectroscopy and UV-VIS-NIR reflectance as well as transmission spectroscopy. The results showed that the films synthesized contained 1-3 layers of graphene with the sheet resistance and optical transparency in the ranges of $50-350 \Omega / s q$ and $85-95 \%$, respectively. Thin films of cadmium sulfide (CdS) were deposited on different substrates by chemical bath deposition method to study the optoelectronic behavior at the interface of graphene/CdS. The results showed that transient response time and the magnitude of photocurrent were enhanced by the graphene electrodes. The order of photocurrent merit was found to be CdS/graphene /Glass > CdS/ITO/glass, indicating that the CdS/graphene stack is to be the most efficient electron transport matrix for the separation of photo excited carriers in the CdS films.
\end{abstract}

Keywords: graphene, CVD process, Raman spectroscopy, optical spectroscopy, optical constants, photoconductivity

*Correspondence: ajayati@utnet.utoledo.edu (A. H. Jayatissa), Tel +1(419) 530-8245, Fax: +1 (419) 530-8206 


\section{Introduction}

Graphene, with its carbon atoms arranged in honeycomb lattice $[1,2]$, has proven to be a useful electronic material after its first discovery in 2004 [3] because of its minimum thickness of $0.32 \AA[4,5]$ and unique physical and electronic properties[1-3]. Its ambipolar transistor behavior [6] high intrinsic carrier mobility of 5000 to $20,000 \mathrm{~cm}^{2} /$ V.s for flat-bed transistors [7] and its tenfold improvement in the mobility exceeding $200,000 \mathrm{~cm}^{2} / \mathrm{V}$.s for suspended graphene films [8] have drawn worldwide attention in semi-conductor industry. In addition to high carrier mobility, graphene based thin film transistors (TFTs) also exhibit large critical current densities $\left(\sim 2 \times 10^{8} \mathrm{~A} / \mathrm{cm}^{2}\right)$ [9], high saturation velocity $\left(\sim 5 \times 10^{7} \mathrm{~cm} / \mathrm{s}\right)$ and very high cutoff frequency up to $100 \mathrm{GHz}$ [10]. Recently, thermally reduced graphene oxide and CNT based devices have been reported to have a strong absorption behavior in the near infrared (NIR) region $[11,12]$. This unusual absorption behavior may provide a pathway to use graphene as an optoelectronic material for infrared remote sensors [13] for the utilization of NIR light in solar-cells and for the successful manifestation of ultrafast optical devices [14-17]. In addition to its superiority over other electronic materials, graphene has also been investigated as a potential candidate for transparent electrodes in photovoltaic devices [18-21] due to its high transparency ( $97.7 \%$ for monolayer) [22] and relatively low sheet resistance ( few hundred $\Omega / s q$ ) $[21,23]$. The characteristics feature of graphene is that even with more than $97 \%$ of transmission, it absorbs rest of the light ( $2.3 \%$ for monolayer) in the visible spectrum without any significant reflection $(<0.1 \%)[1,3]$. Among many different transparent electrodes available in industrial scale, indium tin oxide (ITO) glasses are commonly used in solar cells $[20,22,24,25]$. 
Generally, commercially available ITO glasses show the optical transparency $\sim 90 \%$, reflection $\sim 6 \%$ in visible region $(550 \mathrm{~nm})$, sheet resistance $(<100 \Omega / s q)$ and infinite scalability [25]. Since defect-free graphene transmits more than $95 \%$ of visible light with negligible reflection, it can replace the existing transparent electrode materials, such as ITO and CNTs in optoelectronic devices. Until now, very few researchers have demonstrated the use of graphene as a transparent electrode in optoelectronic devices. X. Wang et al first demonstrated the performance of transparent graphene as electrode in organic solar cell [18]. Q. Su and et al constructed the heterojunction solar cell with PEDOT:PSS and $\mathrm{ZnO}$ as the buffer layer with conductive graphene oxide (GO) and reduced graphene oxide (RGO) films [20]. Deposition of graphene by chemical vapor deposition (CVD) has been investigated to produce large-area graphene layers. It has been reported that a more conductive CVD graphene film with sheet resistance $\sim 230 \Omega / s q$ and a transparency of $\sim 72 \%$ (at around $550 \mathrm{~nm}$ ) showed the performance comparable to ITO based device [23]. In this work, we have synthesized a large area and uniform graphene on a copper foil by CVD method and transferred them onto a different substrates followed by etching of copper substrate. These graphene films have a optical transparency in the range of $85-95 \%$, negligible reflection $(<0.4 \%)$ and low sheet resistance in the range of $300 \pm 25 \Omega / s q$.

Nanostructured semiconductor films based on cadmium compounds (CdS, CdSe, CdTe) have been reported very effective for their use in thin-film solar cells with the efficiencies up to $\sim 20 \%$ [26-28]. Recent reports have shown that the semiconductor quantum dots based on cadmium compounds can improve photovoltaic efficiency by generating multiple charge carriers under photo-excitation [29-31]. However, the major impediment in cadmium based 
semiconductors is the fast recombination of charge carriers generated by photo-excitation process [26-31]. In order to enhance the photovoltaic conversion process, it is essential to effectively retard the recombination of electron-hole pairs [32]. One way to minimize recombination process is that the coating of an efficient electron-transport material, such as conductive polymer films, carbon nanotubes (CNTs), graphene [29,32-34]. Graphene is superior to other materials as an electron-transport matrix due to its high 'electrical conductivity and optical transparency ( $95 \%)$. Although graphene/CdS nano-composites synthesized by chemical method have been used by many researchers for photo-catalysis [32,35-37] and optoelectronic devices [38], very few researchers have reported the use of large area graphene as electron transport matrix with cadmium based semiconductor films $[38,39]$. In this work, CVD graphene films were investigated as the transparent electrodes for CdS layers with excellent electron transfer matrix.

\section{Experimental Details}

\subsection{Synthesis, transfer and fabrication of graphene based devices}

Graphene films were grown on a copper foil (Alfa Aesar, $99.9995 \%, 25 \mu \mathrm{m}$ thick) by a CVD method at a temperature of $1100{ }^{\circ} \mathrm{C}$. After graphene films were deposited on a Cu substrate, it was transferred to desired substrate (glass, Au or CdS) by etching of $\mathrm{Cu}$ films with aqueous 1.0 $\mathrm{M} \mathrm{FeCl}_{3}$ at room temperature. The details about the synthesis and the transfer procedure of graphene films have been discussed elsewhere [5, 40-44]. In order to fabricate the devices, a

thin layer of gold $(\sim 100 \mathrm{~nm})$ was coated on the surface of the transferred graphene film by a vacuum evaporation method. The devices were patterned by photolithography process 
followed by the etching of gold with aqueous $\mathrm{KI} / \mathrm{I}_{2}$ solution. Photolithography began with the substrate that was previously cleaned with Isopropyl alcohol (IPA) followed by rinsing with the acetone. The substrates were then coated with a photoresist layer and exposed to a focused beam of UV light through the mask. The exposed areas of the photoresist (PR) were dissolved in a developer solution to create the PR patterns. The CdS thin films were deposited on the gold patterns by chemical bath deposition (CBD) method described in section 2.3. A similar technique was used to deposit CdS thin films on the surface of graphene films. Finally, the PR were removed by rinsing with acetone.

\subsection{Deposition of CdS thin films}

Polycrystalline CdS thin films were deposited on different substrates by a CBD method. The reagents used in $\mathrm{CBD}$ include $0.2 \mathrm{mmol}$ of $\mathrm{Cd}\left(\mathrm{NO}_{3}\right)_{2}, 0.4 \mathrm{mmol}$ thiourea $\left(\mathrm{H}_{2} \mathrm{NCSNH}_{2}\right), 0.9$ mmol of $\mathrm{NH}_{4} \mathrm{NO}_{3}$, and $\mathrm{NH}_{4} \mathrm{OH}(15 \mathrm{ml})$. These chemicals were dissolved 120 of $\mathrm{DI}$ water. The beaker containing the final solution was kept in a hot water bath to maintain the temperature. All the depositions were carried out at an optimal growth temperature of $70{ }^{\circ} \mathrm{C}$. The cleaned substrates were tied up with a Teflon tape and dipped in the above solution. The deposition was carried out for 15 min to make sure that the CdS films are pinhole free. After deposition, the CdS films were washed with DI water to remove the extra solvents and CdS particles on the films and dried under nitrogen ambient at $120^{\circ} \mathrm{C}$.

\subsection{Materials and measurements}


The quality of the graphene films was examined by Raman spectroscopy, scanning electron microscopy (SEM), and optical spectroscopy. The SEM images were obtained using a (scanning electron microscope (Carl Zeiss SMT AG Supra 35VP FE) operating at an accelerating voltage of $15.0 \mathrm{kV}$ and using an in-lens ion annular secondary electron detector. The Raman spectra of graphene were obtained at the room temperature with a Raman spectrometer (Renishaw-Invia) in back scattering geometry with the laser excitation of $632.8 \mathrm{~nm}$ at a power level of $1.7 \mathrm{mV}$. The morphology and the structure of CdS films were also determined by the Xray diffraction (XRD, D/MAX2500) with a $\mathrm{Cu} K \alpha$ radiation. The optical transmittance and reflectance spectra of graphene films, CdS films, ITO and glass substrates were measured with a double beam UV-VIS-NIR spectrophotometer (Shimadzu). Electrical measurements on graphene/CdS films were performed by connecting devices with a HP-4145B semiconductor parameter analyzer and a high impedance multimeter (Keithly, Model-1200) via electrical feedthrough on a test chamber. A laser beam of $520 \mathrm{~nm}(2 \mathrm{~W})$ was used as the IR light source.

\section{Results and Discussion}

\subsection{Structural properties}

Figure 1(a) shows typical Raman spectra of few layer graphene. As reported by many researchers about quality graphene synthesized by CVD process, the Raman peaks appeared corresponding to the $D$ band $\left(\sim 1330 \mathrm{~cm}^{-1}\right), \mathrm{G}$ band $\left(\sim 1580 \mathrm{~cm}^{-1}\right)$ and $2 \mathrm{D}$ band $\left(\sim 2660 \mathrm{~cm}^{-1}\right)$ [45] for films produced in this work. From the typical spectra shown in Fig. 1(a) for few-layer graphene, the following conclusions can be drawn: 
1. The spectra consist of $D$ band, indicating the presence of some defects in the crystal. The intensity of $D$ band relative to $G$ band $\left(I_{D} / I_{G}\right)$ was found to increase with the increase in number of layers in graphene. Although the intensity ratio increased significantly ( 0.16 to $\sim 0.45)$, the change in FWHM was not significant $\left(\sim 30 \mathrm{~cm}^{-1}\right.$ to $\left.\sim 35 \mathrm{~cm}^{-1}\right)$ in going from monolayer to bi- and tri-layer graphene. However, the broadening of $D$ band increased significantly after three layers. The intensity of D band appeared in the Raman spectra for monolayer graphene is either comparable or relatively less than the intensities reported by others $[5,45,46]$. However, its unavoidable to have defects in graphene synthesized on copper substrate through a CVD process. These defects could be associated with the grain boundaries in the copper foils where graphene was initially made. This band also indicates the vacancies and strained hexagonal and non-hexagonal distortions in the film that may have arisen during transfer process [47].

2. The intensity of $\mathrm{G}$ band did not change from monolayer to few layer graphene. The intensity and FWHM of $2 \mathrm{D}$ band relative to $\mathrm{G}$ band $\left(\mathrm{I}_{2 \mathrm{D}} / \mathrm{I}_{\mathrm{G}}\right)$ changed significantly from monolayer to few layer graphene. The intensity of 2D band was twice as strong as G band for monolayer graphene, whereas it was comparable with the intensity of $\mathrm{G}$ band for tri-layer graphene and decreased for further increase in number of layers. On the other hand, FWHM for 2D band increased slowly for graphene layers up to tri-layer and increased significantly afterwards. The broadening of 2D band corresponds to the increase in number of layers. All D, G and 2D bands were shifted towards higher Raman shifts with increasing the number of layers. 
The uniformity of the transparent conducting layer is one of the important characteristics in designing optoelectronic devices. To investigate the uniformity of the layer, a 2 by $2 \mathrm{~cm}^{2}$ area of graphene surface was divided into nearly 230 different grids to identify the uniformity of the surface. Fig. 1(b) and 1 (c) show the distribution of the Full width at half maximum (FWHM) of $2 \mathrm{D}$ band and the intensity ratio of $2 \mathrm{D}$ and $\mathrm{G}$ band. As seen from Fig. 1(b), FWHM mapping of 2D band was centered $\sim 40 \mathrm{~cm}^{-1}$ with more than $80 \%$ of the data within $35 \pm 20 \mathrm{~cm}^{-1}$, while $I_{2 D} / I_{G}$ ratio mapping was centered $\sim 1.2$ with more than $50 \%$ of the data within $1.1 \pm 0.8$. The mappings indicated that the film mostly consisted of few layer ranging from layers 1-5 [45]. For multilayer graphene, 2D band becomes less Raman active compared with $\mathrm{G}$ band and the broadening of 2D band increases significantly $[5,45]$. Similar measurements were performed for the graphene layer transferred on to $\mathrm{SiO}_{2} / \mathrm{Si}$ substrate. The measurements indicated that the intensity of Raman spectra decreased when the graphene films were transferred to the reflecting surface, such as $\mathrm{SiO}_{2}$ and $\mathrm{SiN}_{\mathrm{x}}$. The decrease in Raman intensity can be attributed to the presence of stronger van der Waals bonding between oxide/nitride surface and graphene films as compared with insulating glass substrate.

\subsection{Optical properties}

Figure 2(a) shows the transmittance of the light in the range $200-1100 \mathrm{~nm}$ for few graphene layers. The optical analysis of thin films has always been a useful method to understand the quality of films, thickness, optical properties, and dielectric constants $[4,5,40]$. The reflectance and the transmittance of the films can also be used to determine the number of layers of graphene on different substrates [4]. For the use of graphene layer as the transparent 
electrodes, one of the most important characteristics is their transmittance behavior in the visible and NIR regions of the spectrum. For 1-3 layers, the transmittance (\%) varied in between $85-95 \%$. As shown in Fig. 2(a), the transmission spectra of indium tin oxide (ITO) is comparable to the few layer graphene on glass substrate. Fig. 2(b) shows the histogram distribution of transmittance (\%) for visible light (wavelength of $550 \mathrm{~nm}$ ) for $2 \mathrm{~cm}^{2}$ graphene films. The film was divided into 200 equal grids and the transmittance of the film was recorded from nearly every grids. It was seen that the transmittance varied between $78-97 \%$, with more than $80 \%$ of the data lying within $85-95 \%$. The slight decrease in the transmittance for monolayer graphene compared with theoretical value $\left(T=(1+0.5 v \alpha)^{-2} \sim(1-\right.$ $v \alpha) \sim 97.7 \%)[3,22]$ can be attributed to the contamination and mechanical defects produced during the transfer process. The mechanical defects of graphene can be generated during transfer process from Cu foil to the desired substrate and this effect can be greatly reduced by synthesizing graphene from transfer-free procedure as explained in detail elsewhere [43].

Another remarkable feature of graphene is that it only reflects less than $1 \%$ (ideally $<0.1$ \%) [22] of the light in the visible region. Thus, even with the transmittance of more than $97 \%$, it absorbs around $2-3 \%$ of the light in the visible region, utilizing the rest of the light for the generation of charge carriers. However, the reflectance of graphene increases linearly from monolayer to few-layer graphene. To understand the reflection of light from graphene, reflectance spectra from bi-layer substrate $\left(\mathrm{SiO}_{2} / \mathrm{Si}\right)$ and tri-layer substrate (graphene $/ \mathrm{SiO}_{2} / \mathrm{Si}$ ) were measured. The reflectance spectra were investigated in the spectral range of 300-1100 nm. Popular approach for thin film, Fresnel approach, was employed to theoretically estimate the reflectance of graphene on silicon wafer $[4,53]$. The Si layer was assumed to be semi-infinite 
for the theoretical estimation so that the light ray reflected from the bottom of silicon surface was negligible. This was a reasonable assumption as the thickness of silicon was about 1000 times larger than silicon dioxide and about half a million times larger than the thickness of graphene film. According to the Fresnel approach, the reflected light intensity can be obtained as $[4,5,53]$,

$$
I\left(n_{1}\right)=\left|\frac{\left(r_{1} e^{i\left(\phi_{1}+\phi_{2}\right)}+r_{2} e^{-i\left(\phi_{1}-\phi_{2}\right)}+r_{3} e^{-i\left(\phi_{1}+\phi_{2}\right)}+r_{1} r_{2} r_{3} e^{i\left(\phi_{1}-\phi_{2}\right)}\right.}{\left(e^{i\left(\phi_{1}+\phi_{2}\right)}+r_{1} r_{2} e^{-i\left(\phi_{1}-\phi_{2}\right)}+r_{1} r_{3} e^{-i\left(\phi_{1}+\phi_{2}\right)}+r_{2} r_{3} e^{i\left(\phi_{1}-\phi_{2}\right)}\right)}\right|^{2} .
$$

Here, the relative indices of refraction $r_{1}, r_{2}$ and $r_{3}$ and the phase shift due to the changes in the optical path $\phi_{1}$ and $\phi_{2}$ are defined as below.

$$
\left.\begin{array}{c}
r_{1}=\frac{n_{0}-n_{1}}{n_{0}+n_{1}} \\
r_{2}=\frac{n_{1}-n_{2}}{n_{1}+n_{2}} \\
r_{3}=\frac{n_{2}-n_{3}}{n_{2}+n_{3}}
\end{array}\right\} .
$$

Here, $d_{1}$ and $d_{2}$ are the thicknesses of graphene and $\mathrm{SiO}_{2}$, respectively. The values of the refractive indices of silicon dioxide $\left(n_{2}\right)$ and silicon $\left(n_{3}\right)$ were extracted from different literature $[4,5,53]$ while the refractive index of graphene was varied around $2.6-1.3 i[4]$ as the refractive index of bulk graphite. The refractive index of air was denoted by $n_{0}$. For normal incidence, its value was taken to be one. From the reflected light intensity, contrast spectra was calculated as 
the relative intensity of reflected light in the presence $\left(n_{1} \neq 1\right)$ and absence $\left(n_{1}=1\right)$ of graphene as given by the following relation [5],

$$
C=\frac{I\left(n_{1}=1\right)-I\left(n_{1}\right)}{I\left(n_{1}=1\right)} .
$$

Figure 2(c) shows the contrast spectra obtained from Fresnel approach for different thicknesses of graphene sheets along with the comparison of the contrast spectrum (Figure in the inset) for monolayer graphene obtained from the experimental and the theoretical approach. The contrast spectrum from monolayer graphene had a peak centered at around 570 $\mathrm{nm}$, which made the graphene surface visible. The intensity of the contrast spectrum for monolayer graphene obtained from experimental data was smaller than the simulated one when the refractive index of graphene was assumed to be the same as graphite (2.6-1.3i) [4]. With the increase in number of layers, the peak shifted towards a higher wavelength and the intensity of the peak increased. It was seen from the inset of Fig. 2(c) that the contrast spectrum obtained from Fresnel approach was higher than experimental data for all wavelengths studied. This difference can be attributed to the fact that the theory overestimates the contrast spectrum as it assumes the normal incidence of light on the graphene surface. The slight deviation of light from normal incidence changes the phase of light at the interface affecting the contrast.

To determine the effective band gap energy of CdS/Gr stack, the optical transmittance of $\mathrm{CdS} / \mathrm{Gr} /$ Glass and CdS/Glass surfaces were measured in the range of $200-1100 \mathrm{~nm}$. The transmittance data were normalized to $T_{\text {Normalized }}=100 \%$ in the transparent region $\left(h v=E_{g}\right)$ to 
neglect the effect of Fresnel reflection and scattering losses in the expression for absorption coefficient $\alpha(h v)[54]$

$$
\alpha(h v)=-\frac{1}{d} \ln \left(T_{\text {Normalized }}(h v)\right)
$$

The thicknesses of graphene layer and CdS thin films were estimated from a simple mathematical modeling based on Fresnel approach and experimental data for reflectance (R) and transmittance $(T)$. As shown in Fig. 2(d), the plot of $(\alpha h v)^{2}$ was extrapolated using a linear relationship to $(\alpha h v)^{2}=0$ to identify the direct band gap of $2.40 \pm 0.01 \mathrm{eV}$ for CdS thin films. The value agrees well with the values reported in different literature for the CBD grown CdS films $[34,48]$. The deposition of CdS thin film in different substrate (CdS/Gr/Glass and CdS/Glass) did not produce any significant difference in the value of band gap energy evaluated by this procedure.

Figure 3 shows the SEM images of CdS films deposited by LBD method for 2 min (a), 5 min (b), and $10 \mathrm{~min}(\mathrm{c})$. The inset in Fig. 3(a) shows the particle size distribution of CdS nanoparticles on the substrate. Although, the average sizes of the particles were around $25 \pm 10 \mathrm{~nm}$, the distribution of the particle sizes spread out uniformly from $20-30 \mathrm{~nm}$. The deposition of the film on the substrate for around 10-15 min caused the agglomeration of particles on top of other particles, which were already deposited on the substrate (Fig. 3(c)). We have characterized numerous CdS samples deposited by CBD methods by SEM measurements and found that the deposition should be carried out $10 \mathrm{~min}$ or higher to obtain a pin-hole free films. Thus, all the films investigated in this paper were deposited for $15 \mathrm{~min}$. The average film thickness of $65 \mathrm{~nm}$ was obtained from atomic force microscopy (AFM) method. 
Figure 4(a) shows the typical Raman spectra of the as-prepared CdS thin films. The spectra were recorded over a range of $200-1500 \mathrm{~cm}^{-1}$. Three distinct peaks were observed at around 310, 609 and $918 \mathrm{~cm}^{-1}$. These peaks can be compared, respectively, with the first, second and third-overtones of the longitudinal optical phonon (1LO, 2LO and 3LO) modes of CdS as reported by many researchers for CdS films deposited by different methods $[48,49]$. The Raman spectra of CdS films strongly depend on the grain size, thickness and the crystallinity of the films. The FWHM of the Raman peak associated with $1 \mathrm{LO}$ was found to be around $23 \mathrm{~cm}^{-1}$, whereas FWHM of peak associated with $2 \mathrm{LO}$ and $3 \mathrm{LO}$ phonon modes were around 53 and 82 $\mathrm{cm}^{-1}$. The intensity of peak associated with 1 LO phonon modes was around 2 times stronger than that of $2 \mathrm{LO}$ and around 5 times stronger than that of $3 \mathrm{LO}$ phonon modes. It has been reported that the Raman shift around $310 \mathrm{~cm}^{-1}, 600 \mathrm{~cm}^{-1}, 900 \mathrm{~cm}^{-1}$ and $1200 \mathrm{~cm}^{-1}$ corresponded to the 1LO, 2LO, 3LO and $4 \mathrm{LO}$ phonons as the characteristics of CdS thin film with better crystallinity [48]. Raman peaks associated with the first three overtones of longitudinal optical phonon modes were strong and visible, whereas the peak associated with 4LO phonons was not seen in the spectra shown in Fig. 4(a). However, the FWHM of 1 LO and 2LO and 3LO peaks obtained from the spectra shown in Fig. 4(a) was in the low end of the range $\left(20-120 \mathrm{~cm}^{-1}\right)$ for FWHM reported for cubic CBD-CdS film $[48,50,51]$ indicating that the CdS films deposited in this work had better crystallinity. When the CdS thin films were deposited on graphene films by CBD method, we did not observe any significant difference in the peak location and intensity for Raman spectra from CdS/Gr/glass and CdS/glass stacks in the range $200-1250 \mathrm{~cm}^{-1}$. However, the intensity ratio of $D$ to $G$ band $\left(I_{D} / I_{G}\right)$ increased by around $10 \%$ after the deposition of CdS on graphene surface. The peak location for $\mathrm{G}$ band shifted from $1576 \mathrm{~cm}^{-1}$ to $1550 \mathrm{~cm}^{-1}$. 
Similarly, 2D peak shifted from $2650 \mathrm{~cm}^{-1}$ to $2630 \mathrm{~cm}^{-1}$. The relative increase in the intensity of D band could be ascribed to the additional mechanical (strained hexagonal/non-hexagonal) distortion in the film due to the deposition of CdS. The downward shifting of $\mathrm{G}$ and $2 \mathrm{D}$ band might have occurred due to the increase in the number of layers in the solid state [38].

Figure 4(b) shows the XRD patterns of the CdS thin films deposited on the Gr/Glass substrate. The diffraction patterns show an intense peak at around $26.8^{\circ}$ with FWHM of $2.0^{\circ}$. In addition to the peak at $26.8^{\circ}$, two small peaks at $44.0^{\circ}$ and $52.1^{\circ}$ were also observed with the FWHM of $1.2^{0}$ and $1.8^{0}$, respectively. The peak at $26.8^{0}$ corresponds to the (111) plane, whereas the peaks at $44.0^{\circ}$ and $52.1^{\circ}$ correspond to (220) and (311) reflection planes, respectively [48]. The XRD patterns of the CdS films deposited in this work indicated that the films had a cubic structure with the preferential orientation normal to the (111) direction (JCPDS card 080-0019) [52].

\subsection{Electrical properties}

Figure 5 shows the layer combinations used for electrical characterization in this study. The industrial uses of graphene as transparent electrodes in optoelectronic devices require that the thin film characteristics of graphene (sheet resistance $\left(R_{s}\right)$, carrier concentration $(N)$ and mobility $(\mu))$ should be comparable or better than the common transparent electrodes, such as ITO or CNTs films $[21,23,24]$. We consider primarily the CdS/Gr and CdS/Gr junctions in I-V measurements. A two-point probe method was employed to determine the sheet resistance of graphene transferred to glass substrate. The films synthesized and transferred to different substrates at different times showed $R_{s}$ of around $300 \pm 25 \Omega / s q$. Although there was a 
variation in the sheet resistance by a large degree, more than $10 \%$ of the data were in the range of $50-250 \Omega / s q$ with the optical transmittance in the range $80-90 \%(550 \mathrm{~nm})$, bringing graphene a potential candidate as the transparent electrode in optoelectronic industry. Other thin film characteristics, such as the carrier concentration and carrier mobility were also obtained for graphene films from the graphene based field effect transistors. Due to the unintentional doping by PMMA (used to transfer graphene from $\mathrm{Cu}$ foil to desired substrate), water and oxygen molecules from atmosphere, GFET showed a weak p-type conducting behavior with carrier concentration $(N) \sim 10^{11} \mathrm{~cm}^{-2}$ and hole mobility $(\mu)$ in the range $100-350 \mathrm{~cm}^{2} / V s$. Although the sheet resistance of graphene films was somewhat higher than current transparent electrodes in the industrial scale, it can be significantly lowered with impurity $\mathrm{n}$-type doping (example nitrogen) without changing its transmittance behavior [21].

\subsection{Photoresponse characteristics}

Figure $6(\mathrm{a})$ and $6(\mathrm{~b})$ show the current - voltage $(\mathrm{I}-\mathrm{V})$ characteristics of $\mathrm{CdS}$ thin films with and without using graphene as a transparent electrode (device $a$ and $b$ ). In this measurement a single layer graphene was used because it has the highest optical transmittance. Both the curves were taken under dark and under the illumination of white light. For small bias voltages, the I-V curves for both the devices were linear, indicating the ohmic contact between Au and $\mathrm{CdS}$, and $\mathrm{Au}$ and Graphene films. When the devices were characterized in dark, the current from device (a) was negligible (order of $10^{-9} \mathrm{~A}$ ), indicating the generation of very small current from CdS films for small bias voltages $(<1.0 \mathrm{~V})$. However, the current was drastically increased when the light was irradiated for both the cases. The magnitude of current from device (b) was 
a factor of four times larger than device (a). The substantial increase in current for CdS/Graphene/Glass stacks as compared with CdS/ITO substrate stack can be attributed to the enhanced conductivity of CdS film with graphene. It also indicated that the photo-induced electron hole pairs were effectively separated by graphene films than ITO glass. The nonlinearity of I-V curve in Fig.6(a) is primarily as due to the asymmetry of electrodes configuration hence different off-set voltages at both front and back electrode/semiconductor $(\mathrm{Au} / \mathrm{CdS}$ and $\mathrm{CdS} / \mathrm{ITO})$ junctions [55].

Figure $7(a)$ shows the spectral response of CdS thin films and single layer graphene. In case of CdS film, graphene was used as a transparent electrode. In both cases, the device consisted of the sensing channel with an area of around $24 \mathrm{~mm}^{2}$ ( $6 \mathrm{~mm}$ long, $4 \mathrm{~mm}$ wide). The spectral response for CdS film indicated that the maximum response of the device was at around $500-550 \mathrm{~nm}$ corresponding to $2.25-2.48 \mathrm{eV}$. The value of optical band gap energy determined by the transmittance of CdS/Gr stack was also found in the same range ( $2.40 \mathrm{eV})$. On the other hand, the spectral response of bare graphene as a sensing channel was around 10 times weaker than $\mathrm{CdS} / \mathrm{Gr}$ stack. When the device was illuminated by the light, the device showed a small response at $720-780 \mathrm{~nm}$ range indicating the graphene has some absorption in this region. This spectral response was measured using a monochromatic and calibrated light source (xenon lamp). There are two important remarks from this experiment. In one hand, the use of graphene as a transparent electrode does not change the effective band-gap of CdS film. On the other hand, graphene may absorb light that would pass through the CdS thin film although the effect of inducing electrons and holes is negligible. 
Figures $7(\mathrm{~b})$ and $7(\mathrm{c})$ show the typical time response characteristics of photo-conducting channel based on CdS/Gr and CdS/ITO structures, respectively. A monochromatic light source was used to provide the input light of wavelength $520 \mathrm{~nm}$. The rise and decay curves of the current can be fitted well by the first order exponential behavior as [56],

$$
\begin{aligned}
& I=I_{0}\left[1-\exp \left(-t / t_{2}\right)\right], \\
& I=I_{0} \exp \left(-t / t_{1}\right) .
\end{aligned}
$$

Here, Eqn. (6) represents the response of the device under the illumination of light and Eqn. (7) represents the recovery of the device under dark. Current $(I)$ is measured as a function of time $(t) . I_{0}$ is the steady state current. $t_{1}$ and $t_{2}$ are the time constants representing the photocurrent rise and fall, respectively. The mean time constants were $90 \mu \mathrm{s}$ and $160 \mu \mathrm{s}$ from CdS/Gr/Glass channel for rise and fall of the photocurrents, respectively. Whereas, the respective values were $430 \mu \mathrm{s}$ and $700 \mu \mathrm{s}$ from CdS/ITO channel. As seen in Fig. 7(b) and 7(c), the relative rise in the current (under the illumination of monochromatic light of wavelength $520 \mathrm{~nm}$ ) was higher for CdS/Gr/Glass surface by a factor of four as compared with CdS/ITO surface. This can be attributed to the fact that graphene effectively accepts photo-generated electrons from the conduction band of CdS to their own conduction band before being recombined hence improved the charge separation of photo-generated electrons and holes [57].

\section{Conclusions}

In summary, we have successfully demonstrated the large scale CVD grown graphene as a potential candidate for transparent electrode in optoelectronic devices. Surface characterization of the graphene films transferred to glass substrate showed that more than 80 
$\%$ of the film area contained 1-3 layers of graphene without any significant breakage and damages. The films showed high optical transparency in the range of $85-95 \%$ at $550 \mathrm{~nm}$. Thin film characterization showed that a carrier mobility of $100-350 \mathrm{~cm}^{2} / \mathrm{Vs}$, carrier concentration $\sim 10^{11} \mathrm{~cm}^{-2}$ and sheet resistance in the range of $50-300 \Omega / s q$ for more than $80 \%$ of the film area. High optical transparency with few hundred $\Omega / s q$ sheet resistance can surpass the minimum industry standard for transparent electrodes, such as ITO and CNT films. We have also demonstrated graphene as an effective electron-transport matrix for CdS thin films because CdS films have been widely used in photovoltaic industry. The photocurrent generated by $\mathrm{CdS}$ thin films under the irradiation of monochromatic light at $520 \mathrm{~nm}$ showed an increase by a factor of four from CdS/Gr/Glass stack as compared with CdS/ITO stack. In addition, the transient response time of the device was also enhanced by a factor of around five with graphene based devices. Therefore, the use of graphene as transparent electrode in optoelectronic devices can enhance the response behavior by effectively retarding the recombination of photo-generated electron-hole pairs .

Acknowledgements: This research was supported by a grant (Grant number: ECCS 0925783) from National Science Foundation (NSF) of USA. 


\section{References}

1. Geim, A. K.; Novoselov, K. S. Nat Mater 2007, 6, (3), 183-191.

2. Katsnelson, M. I. Materials Today 2007, 10, (1-2), 20-27.

3. Novoselov, K. S.; Geim, A. K.; Morozov, S. V.; Jiang, D.; Katsnelson, M. I.; Grigorieva, I. V.; Dubonos, S. V.; Firsov, A. A. Nature 2005, 438, (7065), 197-200.

4. Ni, Z. H.; Wang, H. M.; Kasim, J.; Fan, H. M.; Yu, T.; Wu, Y. H.; Feng, Y. P.; Shen, Z. X. Nano Letters 2007, 7, (9), 2758-2763.

5. Gautam, M.; Jayatissa, A. H. Materials Science and Engineering: C 2011, 31, (7), 1405-1411.

6. Liang, X.; Fu, Z.; Chou, S. Y. Nano Letters 2007, 7, (12), 3840-3844.

7. Avouris, P. Nano Letters 2010, 10, (11), 4285-4294.

8. Bolotin, K. I.; Sikes, K. J.; Jiang, Z.; Klima, M.; Fudenberg, G.; Hone, J.; Kim, P.; Stormer, H. L. Solid State Communications 2008, 146, (9-10), 351-355.

9. Murali, R.; Yang, Y.; Brenner, K.; Beck, T.; Meindl, J. D. Applied Physics Letters 2009, 94, (24), 243114-3.

10. Lin, Y.-M.; Dimitrakopoulos, C.; Jenkins, K. A.; Farmer, D. B.; Chiu, H.-Y.; Grill, A.; Avouris, P. Science 2010, 327, (5966), 662.

11. Acik, M.; Lee, G.; Mattevi, C.; Chhowalla, M.; Cho, K.; Chabal, Y. J. Nat Mater 2010, 9, (10), 840-845.

12. Pradhan, B.; Setyowati, K.; Liu, H.; Waldeck, D. H.; Chen, J. Nano Letters 2008, 8, (4), 11421146.

13. Lo, C. P.; Quattrochi, D. A.; Luvall, J. C. International Journal of Remote Sensing 1997, 18, (2), 287-304. 
14. Yoshino, K.; Lee, S.; Fujii, A.; Nakayama, H.; Schneider, W.; Naka, A.; Ishikawa, M. Advanced Materials 1999, 11, (16), 1382-1385.

15. Brabec, C. J.; Winder, C.; Sariciftci, N. S.; Hummelen, J. C.; Dhanabalan, A.; van Hal, P. A.; Janssen, R. A. J. Advanced Functional Materials 2002, 12, (10), 709-712.

16. Yao, Y.; Liang, Y.; Shrotriya, V.; Xiao, S.; Yu, L.; Yang, Y. Advanced Materials 2007, 19, (22), 3979-3983.

17. Lai, K. W. C.; Xi, N.; Fung, C. K. M.; Chen, H.; Tarn, T.-J. Applied Physics Letters 2009, 95, (22), 221107-3.

18. Wang, X.; Zhi, L.; Mullen, K. Nano Letters 2007, 8, (1), 323-327.

19. Gomez De Arco, L.; Zhang, Y.; Schlenker, C. W.; Ryu, K.; Thompson, M. E.; Zhou, C. ACS Nano 2010, 4, (5), 2865-2873.

20. Su, Q.; Pang, S.; Alijani, V.; Li, C.; Feng, X.; Müllen, K. Advanced Materials 2009, 21, (31), 3191-3195.

21. Pang, S.; Hernandez, Y.; Feng, X.; Müllen, K. Advanced Materials 2011, 23, (25), 2779-2795.

22. Nair, R. R.; Blake, P.; Grigorenko, A. N.; Novoselov, K. S.; Booth, T. J.; Stauber, T.; Peres, N. M. R.; Geim, A. K. Science 2008, 320, (5881), 1308.

23. De, S.; Coleman, J. N. ACS Nano 2010, 4, (5), 2713-2720.

24. Bae, S.; Kim, H.; Lee, Y.; Xu, X.; Park, J.-S.; Zheng, Y.; Balakrishnan, J.; Lei, T.; Ri Kim, H.; Song, Y. I.; Kim, Y.-J.; Kim, K. S.; Ozyilmaz, B.; Ahn, J.-H.; Hong, B. H.; lijima, S. Nat Nano 2010, 5, (8), 574-578.

25. Ocal, T.; Yusuf, S.; Gulnur, A.; Lutfi, O. Journal of Physics D: Applied Physics 2010, 43, (5), 055402. 
26. Brown, P.; Takechi, K.; Kamat, P. V. The Journal of Physical Chemistry C 2008, 112, (12), 4776-4782.

27. Green, M. A.; Emery, K.; Hishikawa, Y.; Warta, W.; Dunlop, E. D. Progress in Photovoltaics: Research and Applications 2012, 20, (5), 606-614.

28. Pookpanratana, S.; Liu, X.; Paudel, N. R.; Weinhardt, L.; Bar, M.; Zhang, Y.; Ranasinghe, A.; Khan, F.; Blum, M.; Yang, W.; Compaan, A. D.; Heske, C. Applied Physics Letters 2010, 97, (17), 172109-3.

29. Brown, P.; Kamat, P. V. Journal of the American Chemical Society 2008, 130, (28), 88908891.

30. Schaller, R. D.; Agranovich, V. M.; Klimov, V. I. Nat Phys 2005, 1, (3), 189-194.

31. Ellingson, R. J.; Beard, M. C.; Johnson, J. C.; Yu, P.; Micic, O. I.; Nozik, A. J.; Shabaev, A.; Efros, A. L. Nano Letters 2005, 5, (5), 865-871.

32. Jia, L.; Wang, D.-H.; Huang, Y.-X.; Xu, A.-W.; Yu, H.-Q. The Journal of Physical Chemistry C 2011, 115, (23), 11466-11473.

33. Zhang, H.; Zhu, Y. The Journal of Physical Chemistry C 2010, 114, (13), 5822-5826.

34. Qing, H.; Lian, G. Nanotechnology 2004, 15, (12), 1855.

35. Wu, J.; Bai, S.; Shen, X.; Jiang, L. Applied Surface Science 2010, 257, (3), 747-751.

36. Cao, A.; Liu, Z.; Chu, S.; Wu, M.; Ye, Z.; Cai, Z.; Chang, Y.; Wang, S.; Gong, Q.; Liu, Y. Advanced Materials 2010, 22, (1), 103-106.

37. Gao, Z.; Liu, N.; Wu, D.; Tao, W.; Xu, F.; Jiang, K. Applied Surface Science 2012, 258, (7), 2473-2478.

38. Miao, F.; Ruiqing, S.; Hongbing, Z.; Yu, C. Nanotechnology 2010, 21, (7), 075601. 
39. Lee, H.; Heo, K.; Park, J.; Park, Y.; Noh, S.; Kim, K. S.; Lee, C.; Hong, B. H.; Jian, J.; Hong, S. Journal of Materials Chemistry 2012, 22, (17), 8372-8376.

40. Gautam, M.; Jayatissa, A.; Sumanasekera, G. In Synthesis and characterization of transferable graphene by CVD method, 2010; IEEE: pp 1-5.

41. Gautam, M.; Jayatissa, A. H. In Ammonia sensor device using graphene modified with platinum, 2011; IEEE: pp 1-2. DOI: 10.1109/NMDC.2010.5652416

42. Gautam, M.; Jayatissa, A. H. Journal of Applied Physics 2012, 111, (9), 094317-094317-9.

43. Gautam, M.; Jayatissa, A. H. Journal of Applied Physics 2012, 112, (6), 064304-064304-7.

44. Gautam, M.; Jayatissa, A. H. Solid-State Electronics 2012, 78, 159-165. DOI: 10.1016/j.sse.2012.05.059

45. Ferrari, A. C.; Meyer, J. C.; Scardaci, V.; Casiraghi, C.; Lazzeri, M.; Mauri, F.; Piscanec, S.; Jiang, D.; Novoselov, K. S.; Roth, S.; Geim, A. K. Physical Review Letters 2006, 97, (18), 187401.

46. Malard, L. M.; Pimenta, M. A.; Dresselhaus, G.; Dresselhaus, M. S. Physics Reports 2009, 473, (5-6), 51-87.

47. Robin, J.; Ashokreddy, A.; Vijayan, C.; Pradeep, T. Nanotechnology 2011, 22, (16), 165701.

48. Oladeji, I. O.; Chow, L.; Liu, J. R.; Chu, W. K.; Bustamante, A. N. P.; Fredricksen, C.; Schulte, A. F. Thin Solid Films 2000, 359, (2), 154-159.

49. Shen, X.-P.; Yuan, A.-H.; Wang, F.; Hong, J.-M.; Xu, Z. Solid State Communications 2005, 133, (1), 19-22.

50. de Melo, O.; Hernandez, L.; Zelaya-Angel, O.; Lozada-Morales, R.; Becerril, M.; Vasco, E. Applied Physics Letters 1994, 65, (10), 1278-1280. 
51. Zelaya-Angel, O.; Castillo-Alvarado, F. d. L.; Avendaño-López, J.; Escamilla-Esquivel, A.; Contreras-Puente, G.; Lozada-Morales, R.; Torres-Delgado, G. Solid State Communications 1997, 104, (3), 161-166.

52. Moualkia, H.; Hariech, S.; Aida, M. S.; Attaf, N.; Laifa, E. L. Journal of Physics D: Applied Physics 2009, 42, (13), 135404.

53. Blake, P.; Hill, E. W.; Neto, A. H. C.; Novoselov, K. S.; Jiang, D.; Yang, R.; Booth, T. J.; Geim, A. K. Applied Physics Letters 2007, 91, (6), 063124-3.

54. Ahalapitiya, H. J.; Ting, W.; Madhav, G. Journal of Physics D: Applied Physics 2012, 45, (4), 045402.

55. S. Selberherr, H. Stippel, E. Strasser (ED), Simulation of Semiconductor Devices and Processess, Vol. 5 (1993) p. 445

56. Shaoxin, L.; Balaji, P. Nanotechnology 2006, 17, (8), 1843.

57. White, J. C.; Dutta, P. K. The Journal of Physical Chemistry C 2011, 115, (7), 2938-2947. 


\section{Figure Captions}

Figure 1: (a) A typical as-measured Raman spectra of few-layer graphene, (b) histogram distribution of intensity ratio $\left(I_{2 D} / I_{G}\right)$, and (c) FWHM mapping of $2 \mathrm{D}$ band from bare graphene on glass substrate.

Figure 2: (a) Transmittance spectra of graphene layers (layer 1, 3 and 5) transferred onto glass substrate along with the spectra of ITO glass as received, (b) histogram distribution of Transmittance (\%) measured for light with wavelength $550 \mathrm{~nm}$, (c) optical contrast of graphene films with increasing layers, and (d) plot of the plot of $(\alpha h v)^{2}$ as a function of photon energy for the transmittance measurement of CdS/Gr stack.

Figure 3: SEM images of CdS thin films deposited on glass substrate: (a) deposition after 2 min; (b) deposition for $5 \mathrm{~min}$; and (c) deposition for $10 \mathrm{~min}$. The Inset of Fig.(a) is the histogram distribution of the particle size in the film.

Figure 4: (a) A typical as-measured Raman spectra of CdS and (b) XRD patterns of CdS thin films on a glass substrate.

Figure 5: Schematic of the devices fabricated in this work: (a) CdS thin deposited on ITO glass where the top Au electrode was deposited by thermal evaporation technique through a photolithography and lift-off processes, (b) CdS on graphene film, (c) CdS layer between two graphene films; and (d) Graphene film on glass with Au electrodes. 
Figure 6: The current - voltage (I-V) characteristics of (a) Au/CdS/ITO and (b) Au/CdS/Gr under dark and white light of average intensity of $290 \mathrm{~cd}$.

Figure 7: (a) Spectral responses of CdS/Gr/Glass (dash line) and Gr/Glass (solid line), (b) photo response behavior of $\mathrm{CdS} / \mathrm{Gr} / \mathrm{Glass}$ under the illumination of monochromatic light of wavelength $520 \mathrm{~nm}$, and (c) photo response behavior of CdS/ITO/glass under the illumination of monochromatic light of wavelength $520 \mathrm{~nm}$. 


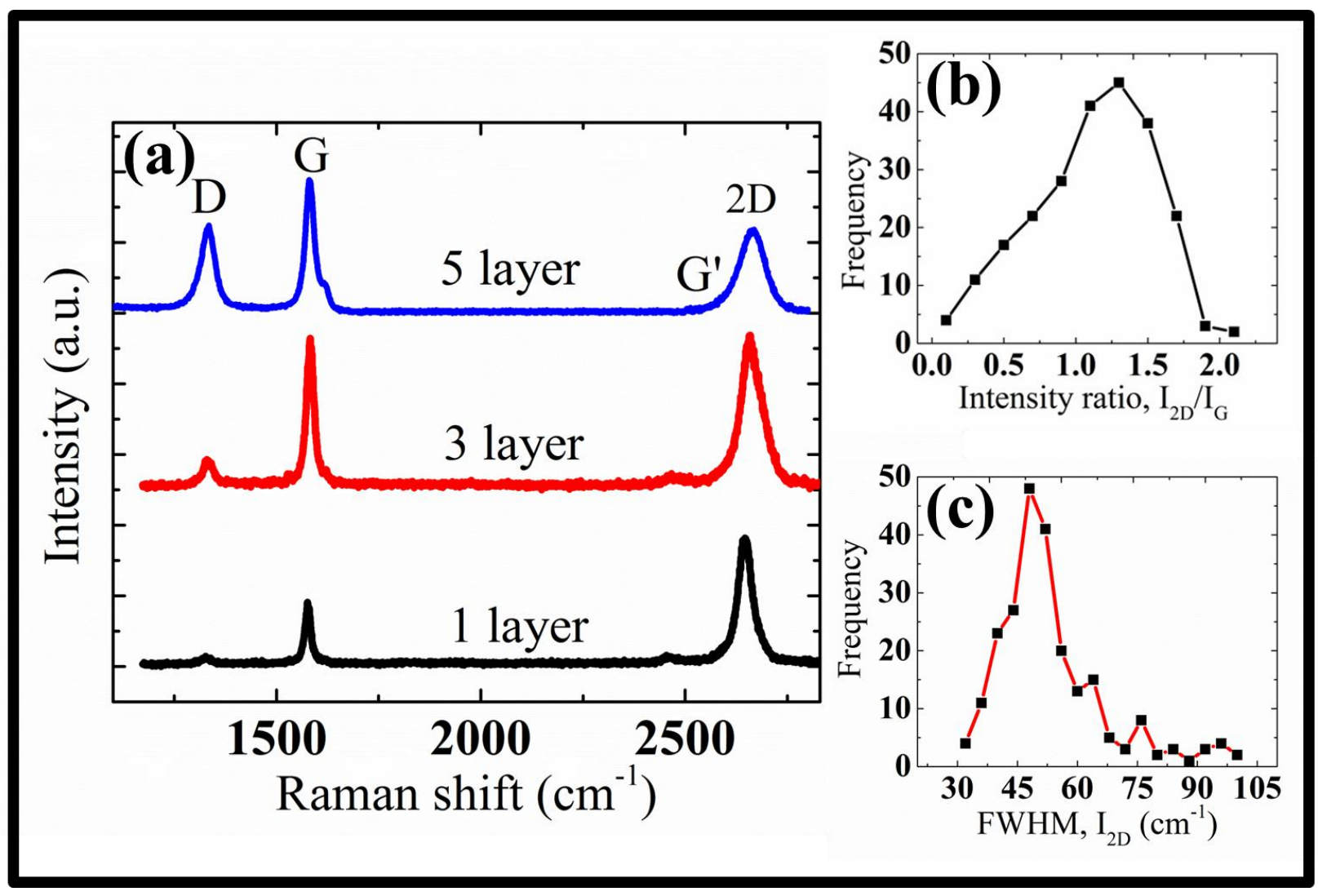

Figure 1 


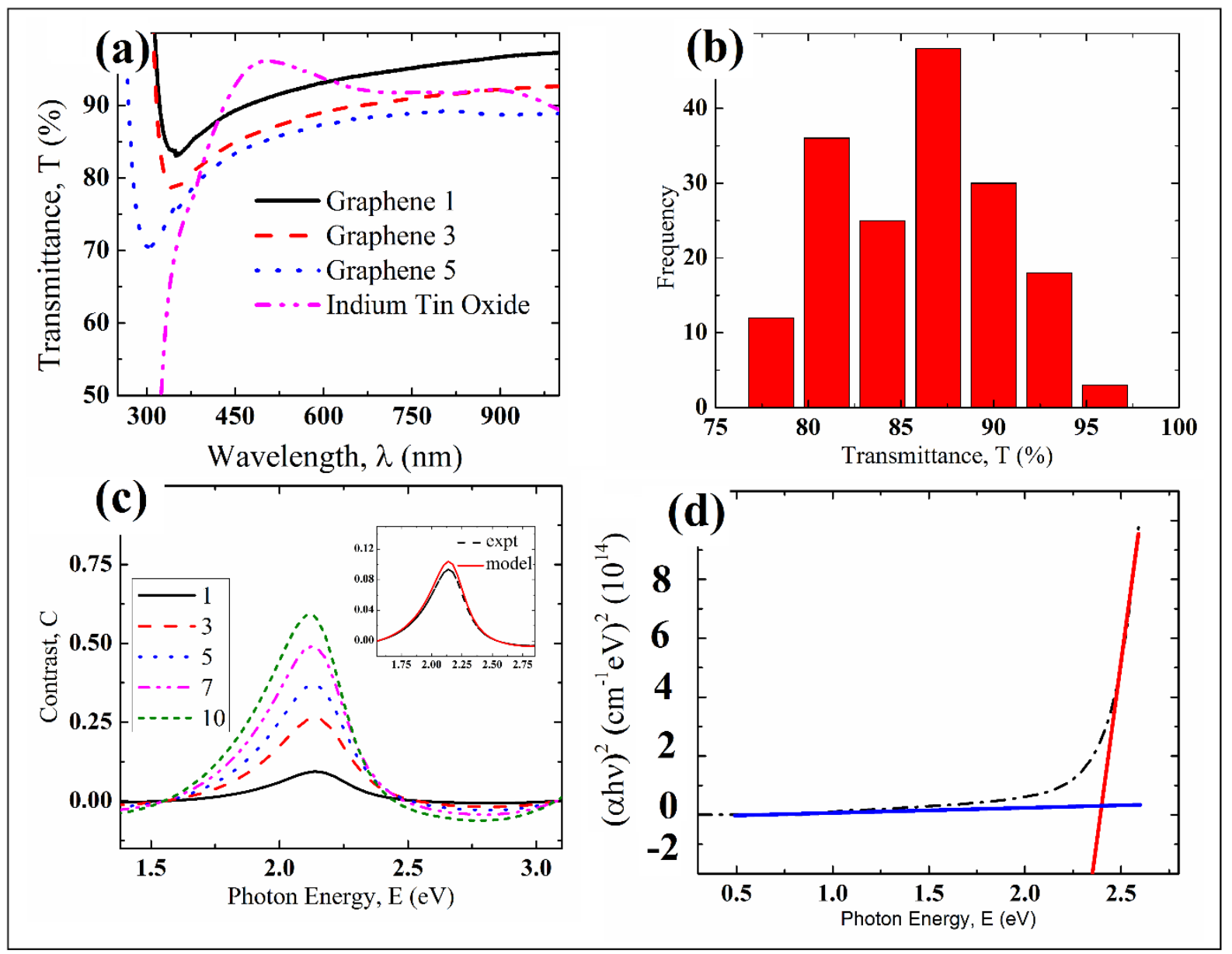

Figure 2 


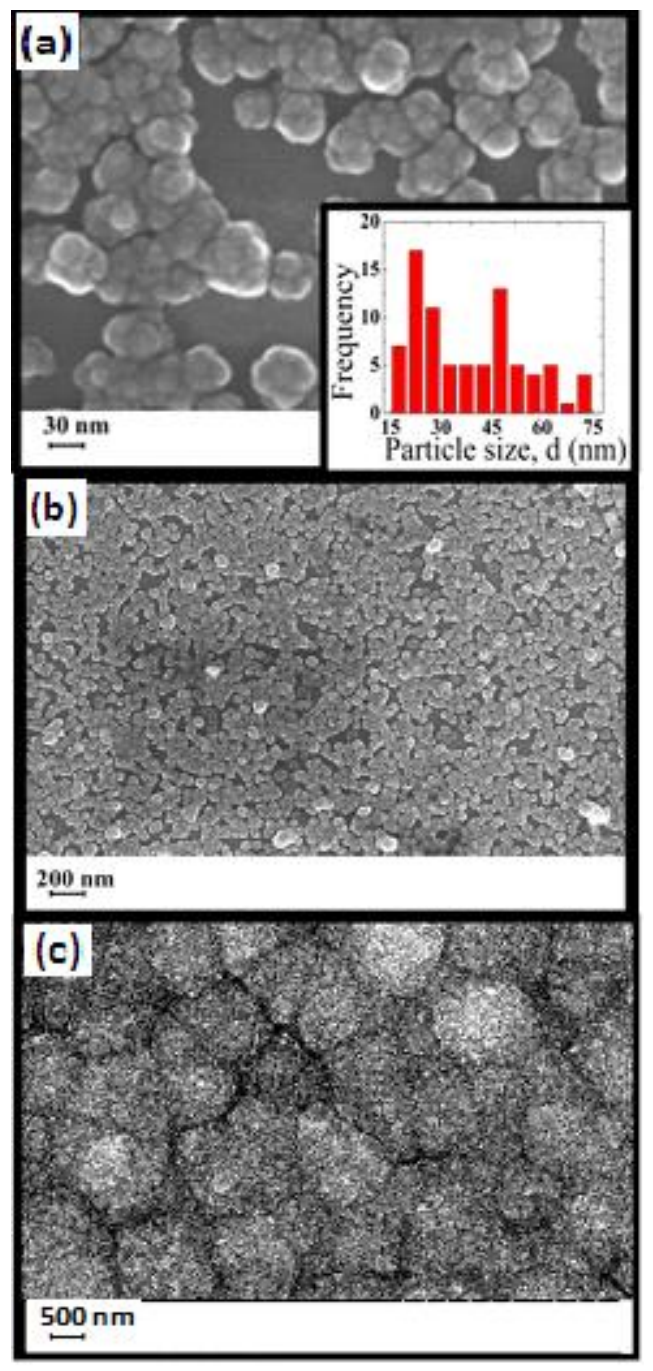

Figure 3 


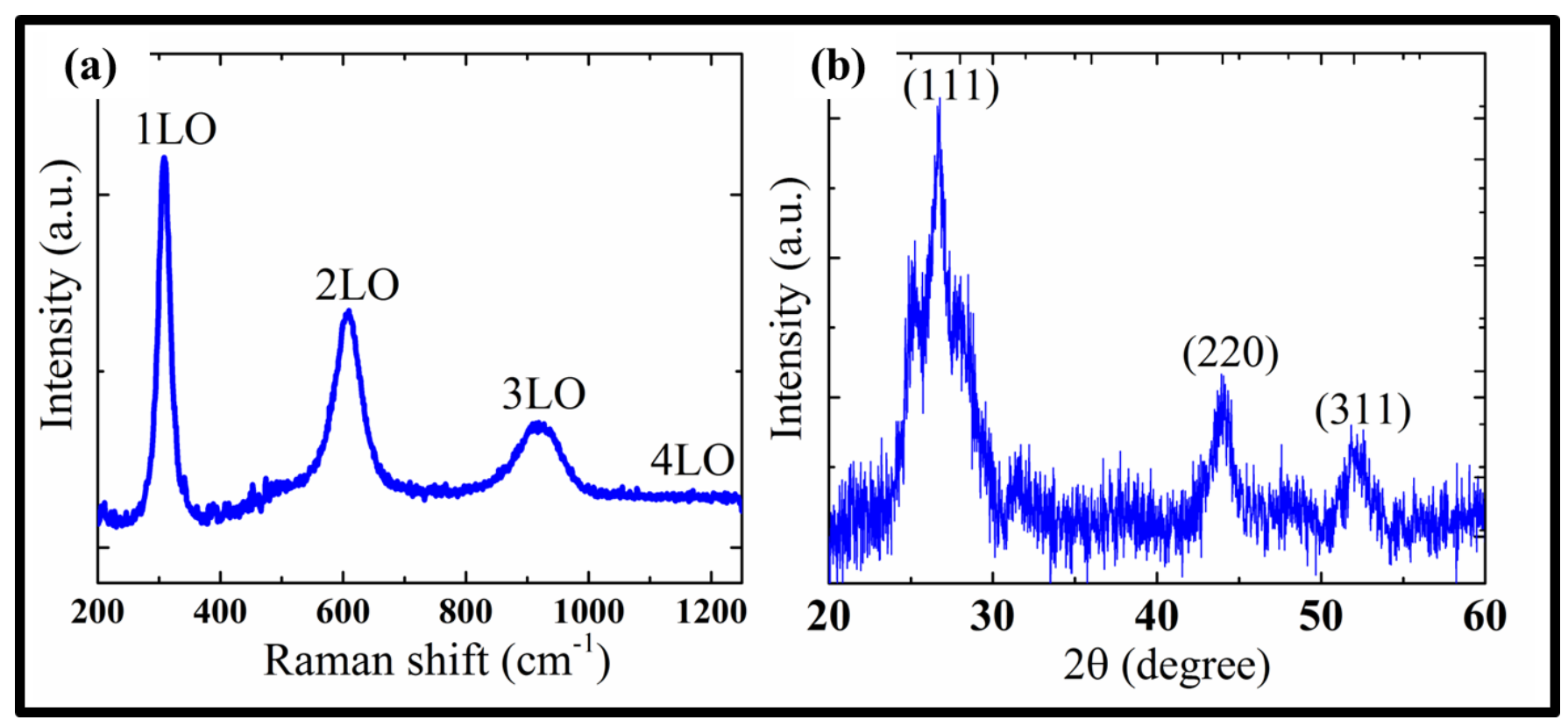

Figure 4 


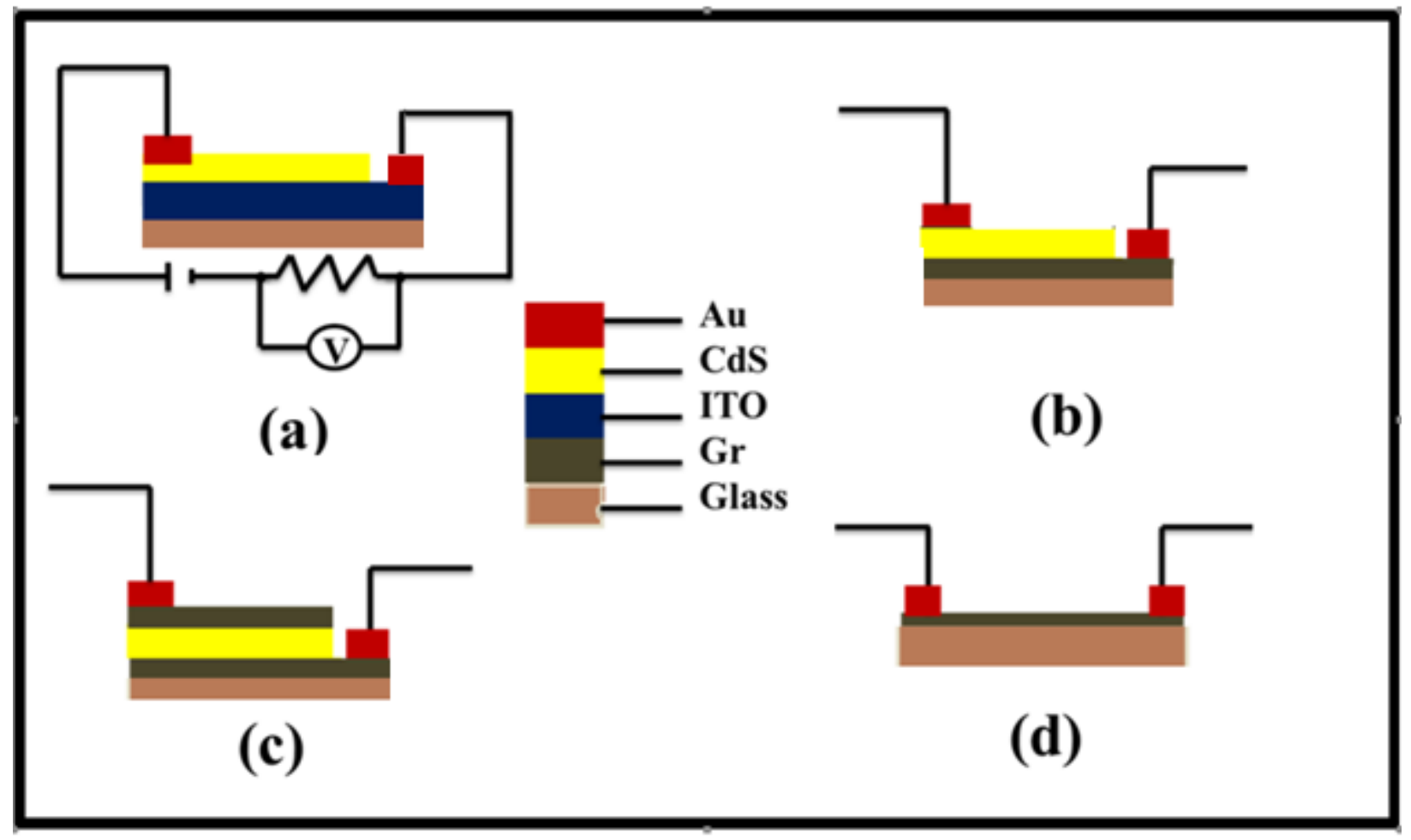

Figure 5 


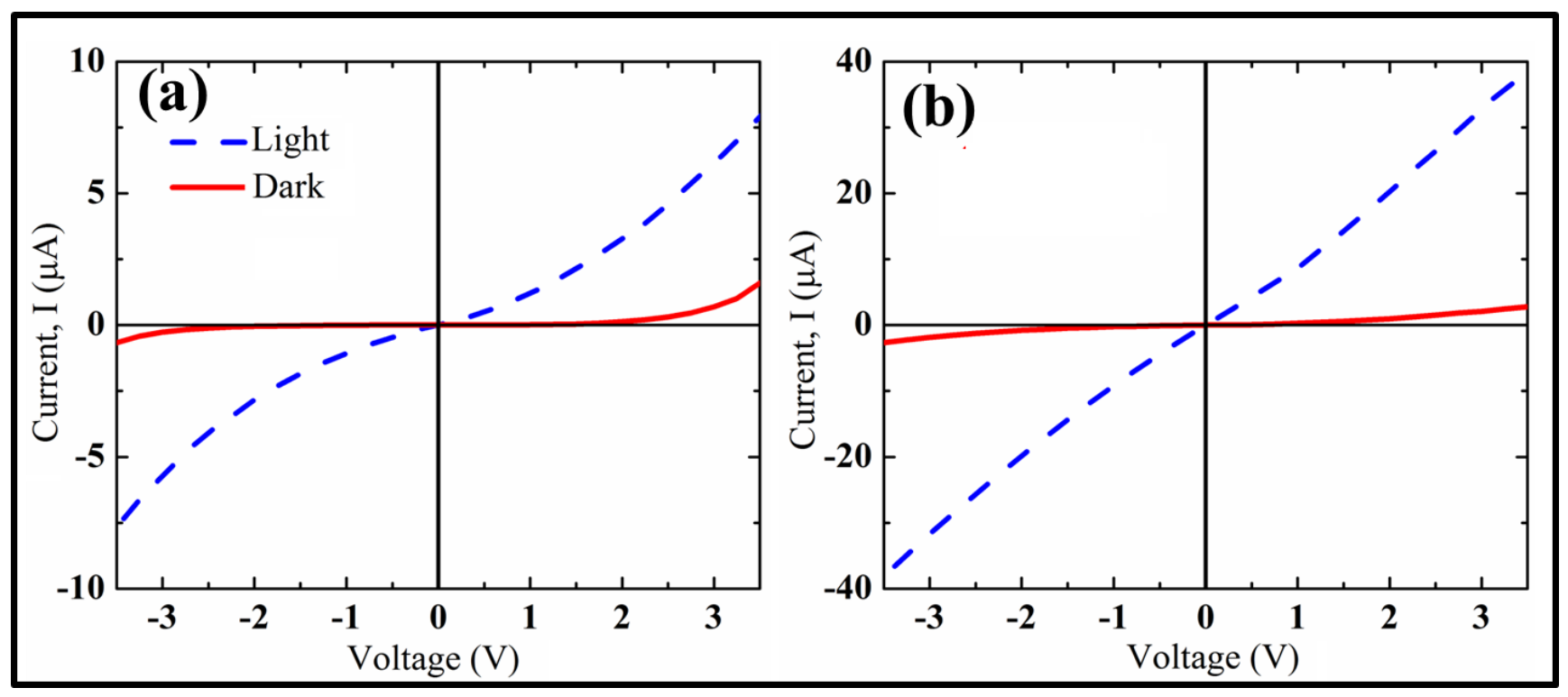

Figure 6 


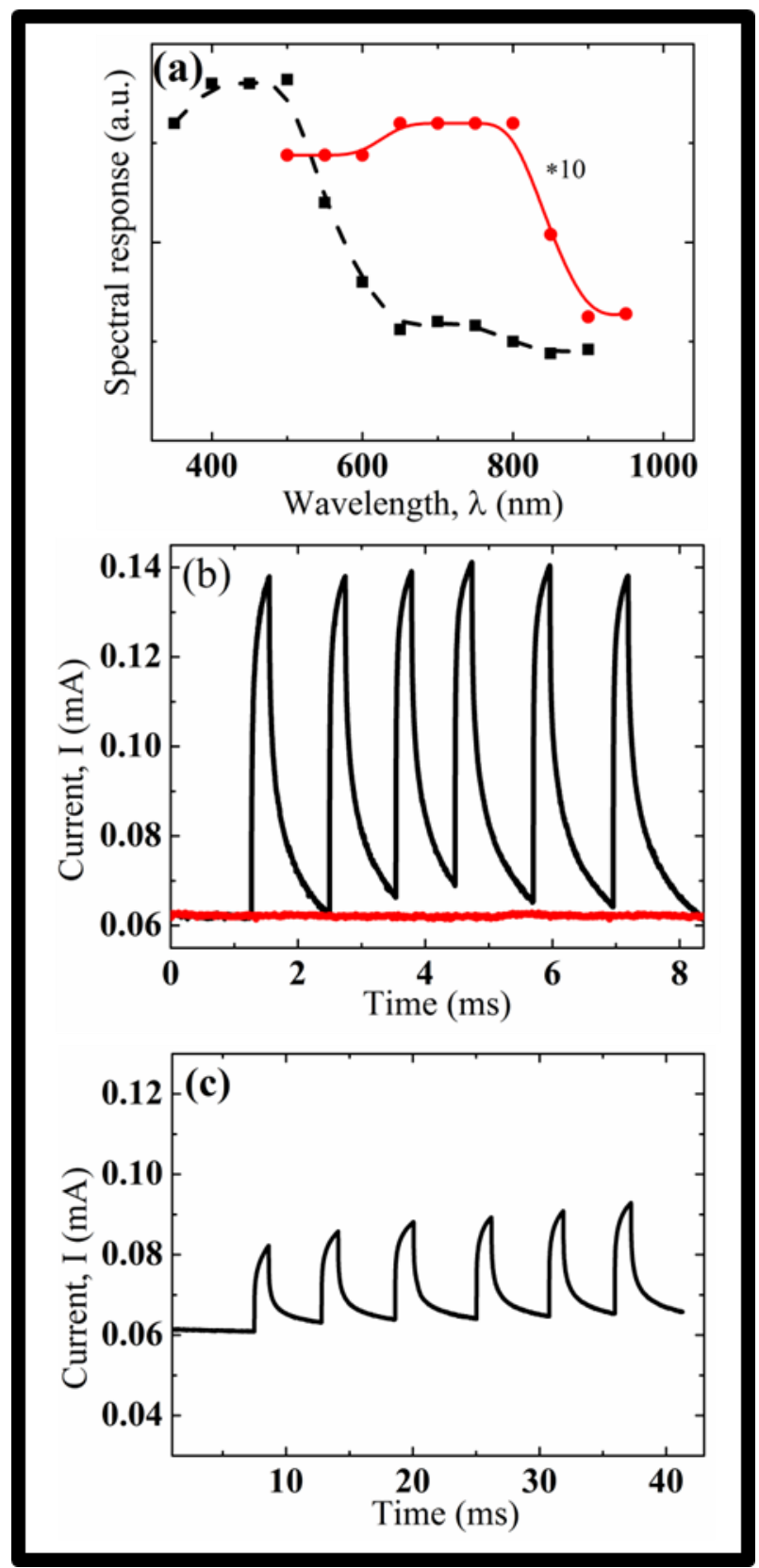

Figure 7 